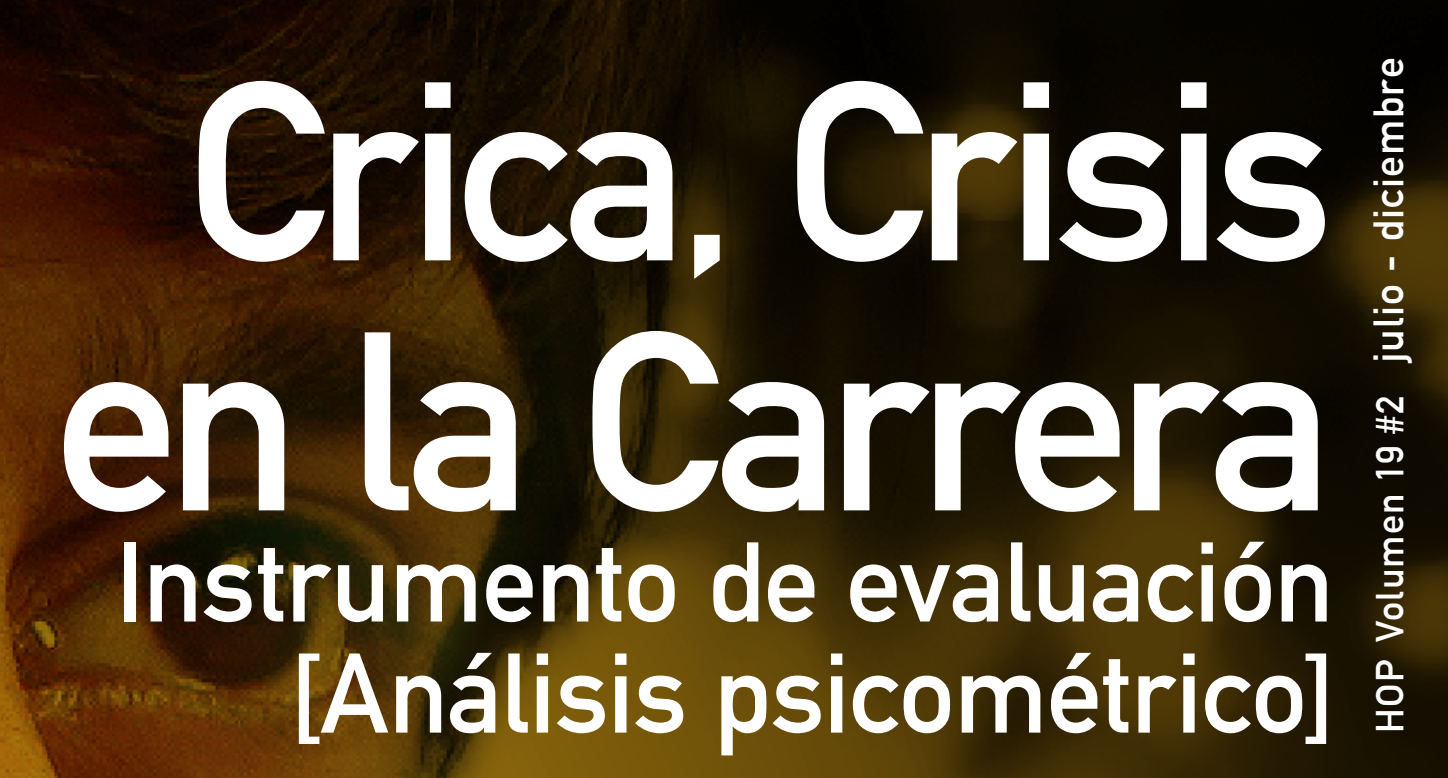

Career crisis evaluation instrument - Crica: [psychometric analysis]

(c) (i)

Elio Leonardo Pardo Jaime Jenni Katherine García Guerrero

\section{[i]}

IBEROAMERICANA

CORPORACIÓN UNIVERSITARIA
HORIZONTES PEDAGÓGICOS ISSN-I: 0123-8264 | e-ISSN: 2500-705X 


\section{HORIZONTES PEDAGÓGICOS}

ID:

0123-8264.hop.19204

\section{Resumen}

Esta investigación hace un análisis psicométrico del instrumento de evaluación de crisis de la carrera (CRICA) en estudiantes de una universidad privada de la ciudad de Bogotá. Para establecer esto se contó con la participación de 137 estudiantes de cinco programas profesionales, 97 hombres y 40 mujeres con edades comprendidas entre los 18 y 34 años (X: 25.68). Dentro de los resultados se encontró un alfa de Cronbach superior a 0.7 (Proyección = 0.700; Motivación = 0.781; Prueba =0.786) y el análisis factorial demostró que el instrumento está dividido en dos factores, compuesto cada uno por cuatro ítems que presenta la misma congruencia teórica propuesta en el momento de su creación; lo cual demuestra que el instrumento es válido y confiable.

\section{Abstract}

The research has as objective to perform a psychometric analysis of the evaluation tool of the crisis of the career (CRICA) in students from a private university in Bogota city. A total of 137 students from five professional programs, 97 men and 40 women aged between 18 and 34 years (X: 25.68) participated. Within the results, a Cronbach"s alpha greater than 0.7 (Projection

$=0.700$; Motivation $=0.781$; Test $=0.786$ ) was

found and the factorial analysis showed that the instrument is divided into two factors, each composed of four items. The same theoretical

congruence proposed at the time of its creation, which shows that the instrument is valid and reliable.
Title: Crica, Career crisis evaluation instrument

Subtitle: [psychometric analysis]

Título: Crica, Crisis en la carrera

Subtítulo: Instrumento de evaluación [Análisis psicométrico]

Alt Title / Título alternativo:

[en]: $\quad$ Psychometric analysis of the caree crisis evaluation instrument - Crica

[es]: $\quad$ Análisis psicométrico del

instrumento de evaluación de crisis de la carrera (CRICA)

Author (s) / Autor (es):

Pardo Jaime, \& García Guerrero

Keywords / Palabras Clave:

[en]: desertion; psychometric analysis; reliability; career crisis validity

[es]: $\quad$ deserción; análisis psicométrico; confiabilidad; validez; crisis de carrera

Submited: 2017-01-12

Acepted: 2017-06-16
Elio Leonardo Pardo Jaime, Psi

\section{Source I Filiacion:}

Corporación Universitaria Iberoamericana

BIO:

Psicologo

City I Ciudad:

Bogotá DC [Co]

e-mail:

epardoj@iberoamericana.edu.co
Jenni Katherine García Guerrero Psi

\section{Source I Filiacion}

Corporación Universitaria Iberoamericana

BIO:

Psicologo

City I Ciudad:

Bogotá DC [Co]

e-mail:

jgarciag@iberoamericana.edu.co

Citar como:

Pardo Jaime, E. L., \& García Guerrero, J. K. (2017). Instrumento de evaluación de crisis en la carrera - Crica: Análisis psicométrico. Horizontes Pedagógicos, issn-l:0123-8264, 19 (2), [pgln]-[pgOut]. Obtenido de: https://revistas. iberoamericana.edu.co/index.php/rhpedagogicos/article/view/1148 


\title{
Crica, Crisis en la carrera Instrumento de Evaluación [Análisis psicométrico]
}

Crica, Career crisis evaluation instrument: [psychometric analysis]

Elio Leonardo Pardo Jaime Jenni Katherine García Guerrero

\section{Deserción estudiantil}

\begin{abstract}
El abandono de la carrera se desarrolla en el marco de la deserción estudiantil; siendo entendida como la pausa de los estudios sin llegar a culminarlos, la cual debe prolongarse por un año o más tiempo (Vásquez Velásquez, Castaño Vélez, Gallón Gómez, \& Gómez Portilla, 2003; MinEducación, 2009; Paramo \& Correa Maya, 1999; Pérez Rodríguez, 2015)를 donde diversas investigaciones han establecido que los factores que tienden a influir en este fenómeno pueden ser personales, familiares, académicos, económicos o la presencia de varios. (Acevedo, Torres, \& Tirado, 2015; Ariza Gasca \& Marín Arias, 2009; Heredia Alarcón, y otros, 2015; Huesca Ramírez \& Castaño Corvo, 2007; Lopera Oquendo, 2008; Muradás Pérez, 2016; Nervi, Rodríguez, \& Osada, 2015; Olave Arias, Rojas García, \& Cisneros Estupiñán, 2013; Vallejo Giraldo, 2009)
\end{abstract}

Una descripción más exacta frente a las causas de la deserción establece cuatro categorías:

Las externas al sistema de educación superior, como las condiciones socioeconómicas del estudiante o grupo familiar. Las propias del sistema de educación superior, como el incremento de las matrículas, la carencia de mecanismos de financiación, las políticas de admisión, el desconocimiento de las metodologías o de la profesión, el ambiente educativo y la ausencia de sentido de pertenencia con la universidad. De orden académico, como los exámenes de admisión, la formación previa, el aprendizaje adquirido, la excesiva orientación teórica y la escasa vinculación con el mundo laboral. La de orden personal, las cuales pueden ser motivacionales, la disonancia con sus expectativas a nivel académico y laboral, la poca madurez emocional y la falta de aptitudes. (Flores López, y otros, 2016)
A raíz de esto, el Ministerio de Educación Nacional (2009) realizó una clasificación de la deserción; la cual se basa en el momento en que el estudiante abandona sus estudios:

Deserción precoz, que se presenta cuando el individuo ha sido admitido por la institución de educación superior pero no se matricula.

Deserción temprana, donde el individuo abandona sus estudios en los primeros semestres del programa.

Deserción tardía, cuando el individuo abandona los estudios en los últimos semestres.

Si bien el fenómeno de la deserción se ha centrado en aquella clasificada como temprana, la cual representa el $\mathbf{7 5 \%}$ del abandono presentado por los estudiantes (Olave Arias, Rojas García, \& Cisneros Estupiñán, 2013), la deserción tardía también es un fenómeno que aqueja a las universidades, y se presenta ante la duda del estudiante por continuar o abandonar sus estudios universitarios en etapas media - avanzada de éstos, fenómeno que es conocido como crisis de carrera. (Pardo Jaime, 2015; MinEducación, 2015) 


\section{Instrumento de evaluación}

Análisis psicométrico]

A partir de lo anterior, Pardo Jaime (2015) diseñó el instrumento de evaluación de Crisis de la Carrera (Crica) el cual evalúa dos factores relacionados con este fenómeno:

$\rightarrow$ Proyección laboral, la cual comprende las ideas de la persona frente al proceso de poder ejercer la profesión.

$\rightarrow$ Motivación para estudiar, la cual evalúa las creencias que tiene el estudiante sobre sus capacidades para afrontar las responsabilidades académicas.

El instrumento cuenta con un alfa de Cronbach de $\mathbf{0 . 7 8 2}$ y dos factores que explican el $\mathbf{6 0 . 4 5 7 \%}$ de la varianza total acumulada en estudiantes de una universidad privada de Bogotá, la cual ofrece sus programas a través de ciclos propedéuticos, confirmando un instrumento válido y confiable para esta población (Pardo Jaime, 2015), no obstante, el instrumento no cuenta con estudios posteriores que permitan corroborar esto, por lo cual la presente investigación pretendió establecer las propiedades psicométricas del instrumento de evaluación de crisis de carrera (Crica).

\section{Proceso metodológico}

El análisis psicométrico del instrumento de evaluación de crisis de carrera (Crica) se desarrolló con $\mathbf{1 3 7}$ estudiantes de cinco programas de pregrado de una universidad privada de la ciudad de Bogotá, que cursaban desde tercer hasta decimo semestre (véase Tabla 2), de los cuales $\mathbf{9 7}$ eran hombres $(\mathbf{7 0 . 8 \%})$ y $\mathbf{4 0}$ mujeres $(\mathbf{2 9 . 2 \%}$ ) con edades comprendidas entre los 18 y 34 años. Esta muestra fue seleccionada de forma no aleatoria debido a que la participación dependió de la autorización otorgada por las facultades.

Para el desarrollo de la presente investigación se realizó la aplicación del instrumento de evaluación de crisis de carrera (Crica) desarrollado por Pardo (2015) el cual cuenta con ocho ítems que evalúan dos factores; proyección laboral; y motivación para estudiar (véase Tabla 1).

Tabla 1 Distribución de ítems del instrumento (Crica)

\begin{tabular}{ccccc|}
\hline Factores & \multicolumn{5}{c|}{ Ítems } \\
\hline Proyección Laboral & 1 & 3 & 6 & 8 \\
Motivación para estudiar & 2 & 4 & 5 & 7
\end{tabular}

Fuente: elaboración propia

En primer lugar, se solicitó a las facultades de la universidad la respectiva autorización para realizar este proceso. Una vez obtenida, se pidió a los participantes la firma de un consentimiento informado; con el fin de garantizar el principio de confidencialidad, junto con el instrumento para que fuera diligenciado. Por último, se realizó la tabulación de las respuestas y se procedió con la ejecución de tres procedimientos para establecer los resultados; el primero, se calculó de coeficiente KMO y la prueba de esfericidad de Bartlett para establecer si el tamaño de la muestra es adecuado para los análisis; segundo, se confirmó la estructura del instrumento a través del análisis factorial; y finalmente, se obtuvo el alfa de Cronbach para determinar la confiabilidad de la prueba.

\section{Normatividad}

Para realizar la presente investigación se tomó en cuenta la Resolución 8430 de 1993, donde se estipula este tipo de trabajos como investigaciones sin riesgo y el artículo 5 de la Ley 1090 de 2006, la cual establece el principio de confidencialidad a través de la firma de un consentimiento informado.

\section{Resultados}

Para realizar el análisis de los datos se utilizó el programa estadístico SPSS versión 22. En primer lugar, se calculó el coeficiente KMO y la prueba de esfericidad de Bartlett; que debía ser mayor a $\mathbf{0 . 7}$ y menor a $\mathbf{0 . 0 0 5}$ respectivamente (Riveros Munévar, Bohórquez Borda, López Castillo, \& Sepúlveda Rodríguez, 2015). De acuerdo al cálculo realizado, la muestra es apropiada para realizar el análisis psicométrico (véase Tabla 2).

Tabla 2 Prueba KMO y esfericidad de Bartlett

\begin{tabular}{lcc}
\multicolumn{3}{c}{ Prueba de KMO y Bartlett } \\
Medida Kaiser-Meyer-Olkin de adecuación de muestreo & $\mathbf{0 . 7 8}$ \\
\hline Prueba de esfericidad de Bartlett & Aprox. Chi-cuadrado & 361.03 \\
& Gl & 28 \\
Sig. & 0 \\
& Fuente: elaboración propia \\
\hline
\end{tabular}

\section{Validez de constructo}

Para establecer la validez de constructo se utilizó el análisis factorial con rotación varimax, debido a que éste nos permite establecer los ítems que componen cada factor, así como los factores que componen la prueba (Hernández Sampieri, Fernández Collado, \& Baptista Lucio, 2010). LoS factores extraídos como resultado del análisis se pueden observar en la Tabla 3.

Estos resultados indican que la prueba cuenta con dos factores que explican el $\mathbf{5 9 . 5 1 5 \%}$ de la varianza total acumulada y cada factor está compuesto por cuatro ítems (véase Tabla 4)

Por último, para confirmar si todos los ítems aportan de manera significativa al instrumento, se establecieron los índices de extracción de cada reactivo (véase Tabla 5) En este apartado se puede evidenciar que el ítem 7 presenta la menor correlación con la prueba, mientras que el resto de ítems mantiene una correlación adecuada (extracción $=0.400$ )

Tabla 3 Factores extraídos por el análisis factorial

\begin{tabular}{|c|c|c|c|}
\hline \multicolumn{2}{|c|}{ Varianza total explicada } & \multicolumn{2}{|c|}{ Sumas de rotación de cargas al cuadrado } \\
\hline Componente & Total & $\%$ de varianza & $\%$ acumulado \\
\hline 1 & 2.712 & 33.896 & 33.896 \\
\hline 2 & 2.05 & 25.619 & 59.515 \\
\hline \multicolumn{4}{|c|}{$\begin{array}{r}\text { Método de extracción: análisis de componentes principale } \\
\text { Fuente: elaboración prop }\end{array}$} \\
\hline
\end{tabular}

Tabla 4 Matriz de componentes rotados del Crica

\begin{tabular}{ccc}
$\begin{array}{c}\text { Matriz de componente } \\
\text { rotado a }\end{array}$ & \multicolumn{3}{c|}{ Componente } \\
& $\mathbf{1}$ & $\mathbf{2}$ \\
Ítem 2 & 0.81 & \\
Ítem 4 & 0.85 & \\
Ítem 5 & 0.84 & \\
Ítem 7 & 0.5 & 0.78 \\
Ítem 1 & & 0.68 \\
Ítem 3 & & 0.84 \\
Ítem 6 & & 0.43 \\
Ítem 8 & &
\end{tabular}

Método de extracción: análisis de componentes principales Método de extracción: Varimax con normalización Kaiser. a) La rotación ha covergido en 3 iteraciones Fuente: elaboración propia 
Tabla 5 Índices de extracción del Crica

\begin{tabular}{|ccc|} 
& $\begin{array}{c}\text { Comunalidades } \\
\text { Inicial }\end{array}$ & Extracción \\
\hline Ítem 1 & 1 & 0.614 \\
Ítem 2 & 1 & 0.671 \\
Ítem 3 & 1 & 0.589 \\
\hline Ítem 4 & 1 & 0.744 \\
Ítem 5 & 1 & 0.737 \\
Ítem 6 & 1 & 0.742 \\
Ítem 7 & 1 & 0.25 \\
Ítem 8 & 1 & 0.416
\end{tabular}

Méto do de extracción: análisis de componentes principales Fuente: elaboración propia

\section{Confiabilidad}

Para establecer la confiabilidad de la prueba, se calculó el alfa de Cronbach de la prueba en general, así como de cada factor, donde se establece como confiable si el valor del alfa es mayor a 0.7. A continuación se muestra el alfa de la prueba, cuyo valor cumple con el requisito de confiabilidad (alfa $=0.786$ ) (véase Tabla 6).

Por otra parte, se evaluó el efecto que tendría en el alfa de Cronbach si alguno de los ítems se eliminara. El estadístico refleja que, al eliminar el ítem 1 y el ítem 8, el alfa aumentaría (ítem 1 =0.804; ítem $7=0.790$ ), lo cual es corroborado al verificar la correlación total de elementos, donde los ítems mencionados son los que presentan los valores más bajos (ítem $1=0.324$; ítem $7=0.300$ ) (véase Tabla 7 ).

Tabla 6. Alfa de Cronbach de la prueba

\begin{tabular}{ccc} 
Alfa de Cronbach & \multicolumn{2}{c}{ Estadísticas de fiabilidad } \\
0.786 & -- basado en elementos estandarizados & $\mathbf{N}^{\circ}$ elementos \\
& 0.8 & 8 \\
& & Fuente: elaboración propia
\end{tabular}

Tabla 7 Alfa de Cronbach si se eliminara uno de los ítems de la prueba

\begin{tabular}{ccccc} 
Ítems & $\begin{array}{c}\text { Media de } \\
\text { escala }\end{array}$ & $\begin{array}{c}\text { Varianza de } \\
\text { escala }\end{array}$ & $\begin{array}{c}\text { Correlación total de } \\
\text { elementos corregida }\end{array}$ & $\begin{array}{c}\text { Alfa de } \\
\text { Cronbach }\end{array}$ \\
\hline Ítem 1 & 13.54 & 18.68 & 0.324 & 0.804 \\
Ítem 2 & 14.57 & 18.662 & 0.579 & 0.749 \\
Ítem 3 & 14.97 & 18.281 & 0.557 & 0.752 \\
Ítem 4 & 14.9 & 17.983 & 0.596 & 0.745 \\
Ítem 5 & 15.01 & 18.956 & 0.62 & 0.746 \\
Ítem 6 & 14.75 & 17.937 & 0.579 & 0.748 \\
Ítem 7 & 14.84 & 20.922 & 0.3 & 0.79 \\
Ítem 8 & 15.44 & 20.085 & 0.494 & 0.765 \\
& & & Fuente: elaboración propia \\
\hline
\end{tabular}

Este mismo proceso se realizó para establecer el alfa de ambos factores. Las Tablas 8 y 9 reflejan los resultados del primer factor; en la primera se demuestra que el alfa presenta un valor adecuado para establecer su confiabilidad (alfa $=0.781$ ) (véase Tabla 8); mientras que la segunda refleja que eliminando el ítem 7, el alfa aumentaría de forma considerable (alfa $7=0.843$ ) lo cual se explica debido a la baja correlación que presenta éste con respecto al factor (correlación $7=$ 0.334) (véase Tabla 9).

Tabla 8 Alfa de Cronbach del factor 1

\begin{tabular}{cc}
\multicolumn{2}{c}{ Estadísticas de fiabilidad } \\
Alfa de Cronbach & $\mathbf{N}^{\circ}$ de elementos \\
\hline 0.781 & 4 \\
& Fuente: elaboración propia \\
\hline
\end{tabular}

Tabla 9 Alfa de Cronbach si se eliminara uno de los ítems del factor uno

\begin{tabular}{ccccc} 
Media de & \multicolumn{3}{c}{ Estadística de total de elemento } \\
escala & $\begin{array}{c}\text { Varianza de } \\
\text { escala }\end{array}$ & $\begin{array}{c}\text { Correlación total } \\
\text { elementos corregida }\end{array}$ & $\begin{array}{c}\text { Alfa de } \\
\text { Cronbach }\end{array}$ \\
\hline Ítem 2 & 5.83 & 4.334 & 0.684 & 0.675 \\
Ítem 4 & 6.17 & 4.023 & 0.679 & 0.676 \\
Ítem 5 & 6.27 & 4.659 & 0.686 & 0.682 \\
Ítem 7 & 6.1 & 5.634 & 0.334 & 0.843 \\
& & & Fuente: elaboración propia
\end{tabular}

Por otra parte, la Tabla 10 evidencia que el valor del alfa del segundo factor es aceptable (alfa =0.700); mientras que, en la Tabla 11 se observa que, eliminando el primer ítem, el alfa aumentaría (alfa $1=0.709)$, aunque éste correlaciona de forma adecuada con el factor (correlación $1=0.418$ )

Tabla 10 Alfa de Cronbach del factor 2

\begin{tabular}{|cc|}
\hline \multicolumn{2}{|c|}{ Estadísticas de fiabilidad } \\
\hline Alfa de Cronbach & $\mathbf{N}^{\circ}$ de elementos \\
\hline 0.7 & 4 \\
& Fuente: elaboración propia \\
\hline
\end{tabular}

Tabla 11 Alfa de Cronbach si se eliminara uno de los ítems del factor dos

\begin{tabular}{|c|c|c|c|c|}
\hline \multicolumn{5}{|c|}{ Estadísticas de total de elemento } \\
\hline & $\begin{array}{l}\text { Media de } \\
\text { escala }\end{array}$ & $\begin{array}{l}\text { Varianza de } \\
\text { escala }\end{array}$ & $\begin{array}{l}\text { Correlación total } \\
\text { elementos corregida }\end{array}$ & $\begin{array}{c}\text { Alfa de } \\
\text { Cronbach }\end{array}$ \\
\hline Ítem 1 & 5.42 & 4.907 & 0.418 & 0.709 \\
\hline Ítem 3 & 6.83 & 5.523 & 0.535 & 0.606 \\
\hline Ítem 6 & 6.61 & 4.945 & 0.664 & 0.519 \\
\hline Ítem 8 & 7.31 & 6.891 & 0.388 & 0.694 \\
\hline
\end{tabular}

Fuente: elaboración propia

\section{Conclusiones}

La deserción es un fenómeno que tiene un gran impacto, no solo en quien ha debido tomaresta decisión por distintos factores (académicos, económicos, personales, entre otros) sino también a la institución de educación superior; ya que éste es uno de los indicadores utilizados para evaluar la calidad de los establecimientos universitarios. (Acevedo, Torres, \& Tirado, 2015; Ariza Gasca \& Marín Arias, 2009; Heredia Alarcón, y otros, 2015; Huesca Ramírez \& Castaño Corvo, 2007; Lopera Oquendo, 2008; MinEducación, 2015; Muradás Pérez, 2016; Nervi, Rodríguez, \& Osada, 2015; Olave Arias, Rojas García, \& Cisneros Estupiñán, 2013; Vallejo Giraldo, 2009)

Si bien existen programas y procesos diseñados para prevenir y controlar la deserción temprana (Olave Arias, Rojas García, \& Cisneros Estupiñán, 2013), las tasas de deserción en los diferentes niveles educativos que al 2014 promedian el 53\%; y que se ha mantenido así desde el 2010 (Orozco Silva, 2016), lo cual refleja que las intervenciones diseñadas para esta problemática no han obtenido un gran impacto.

A partir de lo anterior, Pardo Jaime (2015) diseño un instrumento que permite evaluar las causas por las cuales los estudiantes de semestres medios y altos deciden abandonar sus estudios, considerando aquellos factores que la institución puede tener la capacidad de apoyar y de manejar, y aunque los datos psicométricos de su creación fueron adecuados, no existen estudios posteriores que permitan corroborar estos valores, lo cual motivó la presente investigación.

A la luz de los resultados obtenidos por el análisis factorial se 


\section{Instrumento de evaluación}

\section{Análisis psicométrico]}

encuentra un instrumento dividido en dos factores, compuesto cada uno por cuatro ítems que presenta la misma congruencia teórica propuesta en el momento de su creación: proyección laboral y motivación para estudiar (Pardo Jaime, 2015). También se estableció un alfa de Cronbach superior a 0.7 (proyección = 0.700; motivación = 0.781 ; prueba $=0.786$ ) lo cual confirma que el instrumento es confiable a nivel factorial y como prueba en general.

No obstante, se encontraron ítems que afectan el alfa del instrumento: el ítem 1 y el ítem 7. Al evaluar el ítem 1 se encontró que, el aumento del alfa si éste es eliminado no es significativo dentro del factor (diferencia $=0.009$ ) ni en la totalidad de la prueba (diferencia = 0.018); además, dicho ítem presenta una adecuada correlación en ambos análisis (correlación = 0.418; extracción = 0.614) lo cual indica que el ítem no debe ser eliminado.

Por otra parte, si el ítem 7 es eliminado presenta un aumento significativo en el alfa del factor (diferencia $=0.062$ ); no obstante, el aumento en el total de la prueba no es tan influyente (diferencia = 0.004). Al verificar la correlación con respecto al factor y a la prueba, los resultados muestran que ésta es baja en ambos casos (correlación =0.388; extracción = 0.300); implicando que el comportamiento del ítem difiere con respecto a lo evaluado, lo cual conlleva a la necesidad de ajustarlo o suprimirlo.

Con base en esto, es importante realizar estudios que permitan resolver las dificultades encontradas en los ítems uno y siete, a fin de consolidar un instrumento que aporte a mitigar el fenómeno de la deserción, el cual afecta la calidad de la educación en el país, y aún más, la calidad de vida del estudiante involucrado en ella. De igual forma, se extiende la invitación para realizar estas investigaciones con otras poblaciones, ya que, si bien la muestra seleccionada hace parte de varias facultades y fue adecuada para el análisis, ésta tiende a ser homogénea.

\section{Referencias}

Acevedo, D., Torres, J. D., \& Tirado, D. F. (2015). Análisis de la Deserción Estudiantil en el Programa Ingeniería de Alimentos de la Universidad de Cartagena Durante el Periodo Académico 2009 - 2013. Formación Universitaria, 8(1), 35-42. doi:10.4067/S0718-50062015000100005

Ariza Gasca, S. M., \& Marín Arias, D. A. (2009). Factores Intervinientes en la Deserción Escolar de la Facultad de Psicología: Fundación Universitaria Los Libertadores. Tesis psicológica, 4(1), 72-85. Obtenido de http://publicaciones.libertadores.edu.co/index.php/ TesisPsicologica/article/view/214

Flores López, W. O., Gutiérrez y Restrepo, E., León Corredor, O. L., Sarraipa, J., Pantoja Lima, C., Merino, C., ... Boticario, J. (2016). Centros de Apoyo y Desarrollo Educativo Profesional para la Observación y Disminución de la Deserción Universitaria. Ciencia e Interculturalidad, 18(1), 48-62. doi:10.5377/rci.v18i1.3049

Heredia Alarcón, M., Andía Ticona, M., Ocampo Guabloche, H., Ramos Castillo, J., Rodríguez Caldas, A., Tenorio, C., \& Pardo Ruiz, K. (2015). Deserción Estudiantil en las Carreras de la Ciencia de la Salud en el Perú. Anales de la Facultad de Medicina, 76, 57-61. doi:10.15381/anales.v76i1.10972

Hernández Sampieri, R., Fernández Collado, C., \& Baptista Lucio, P. (2010). Metodología de la Investigación (600 ed.). México D.F. [mx]: McGraw-Hill.

Huesca Ramírez, G. E., \& Castaño Corvo, B. (2007). Causas de la Deserción de Alumnos de Primeros Semestres de una Universidad Privada. REMO, 5(12), 34-39. Obtenido de http://www.alfaguia.org/alfaguia/ files/1319582164causas\%20de\%20desercion\%20en\%20una\%20 universidad\%20privada.pdf
Ley 1090 de 2006, Por la cual se reglamenta el ejercicio de la profesión de psicología, se dicta el código deontológico y bioético y otras disposiciones. (Congreso de la República de Colombia 6 de 9 de 2006). Obtenido de http://www.secretariasenado.gov.co/senado/basedoc/ ley 1090 2006.html

Lopera Oquendo, C. (2008). Determinantes de la Deserción Universitaria en la Facultad de Economía de la Universidad del Rosario. Borradores de Investigación(95), 41. Obtenido de http://repository.urosario.edu. co/bitstream/handle/10336/3740/Borrador Economia 95\%5B1\%5D. pdf? sequence $=8 \&$ is Allowed $=y$

MinEducación. (2009). Deserción Estudiantil en la Educación Superior Colombiana: Metodología de seguimiento, diagnóstico y elementos para su prevención (158 ed.). Bogotá D.C. [co]: MinEducación, Ministerio de Educación Nacional. Obtenido de http://www.mineducacion.gov.co/ sistemasdeinformacion/1735/articles-254702 libro desercion.pdf

MinEducación. (2015). Rutas de Vida: Manual para el acompañamiento en orientación socio ocupacional. Bogotá D.C. [co]: MinEducación, Ministerio de Educación Nacional. Obtenido de https://www. mineducacion.gov. co/1759/articles-356514 recurso.pdf

Muradás Pérez, M. (2016). Diagnóstico de Algunos Factores de Riesgo Asociados a la Deserción Estudiantil de los Alumnos de la Universidad Politécnica del Centro. Perspectivas Docentes, 27(62), 35-39. Obtenido de http://revistas. ujat.mx/index.php/perspectivas/article/view/1307/1051

Nervi, C., Rodríguez, J., \& Osada, J. (2015). Deserción Universitaria Durante el Primer Año de Estudios [carta al director]. FEM, 18(2), 93-93. doi:10.4321/ S2014-98322015000200003

Olave Arias, G., Rojas García, l., \& Cisneros Estupiñán, M. (2013). Deserción Universitaria y Alfabetización Académica. Educación y Educadores, 16(3), 455-471. Obtenido de http://educacionyeducadores.unisabana.edu.co/ index.php/eye/article/view/2726/3352

Orozco Silva, L. E. (2016). Educación Superior en Iberoamérica Informe 2016: Informe nacional: Colombia. Bogotá D.C. [co]: Cinda, Universia. Obtenido de http://www.cinda.cl/wp-content/uploads/2016/11/COLOMBIA-InformeFinal.pdf

Paramo, G. J., \& Correa Maya, C. A. (1999). Deserción Estudiantil Universitaria: Conceptualización. Revista Universidad Eafit, 35(114), 66-78. Obtenido de http://publicaciones.eafit.edu.co/index.php/revista-universidad-eafit/ article/view/1075

Pardo Jaime, E. L. (2015). Creación del Instrumento de Evaluación de Crisis de la Carrera - CRICA [proyecto de practica]. Bogotá D.C. [Co]: Corporación Universitaria Iberoamericana.

Pérez Rodríguez, C. L. (2015). Deserción Universitaria: Una mirada desde la experiencia del Departamento de Atención Psicopedagógica. Dia-logos, 16, 39-47. doi:10.5377/dialogos.v0i16.2514

Resolución N8430 de 1993, Por la cual se establecen las normas científicas, técnicas y administrativas para la investigación en salud (Ministerio de Salud 4 de 10 de 1993). Obtenido de https://www.minsalud.gov.co/sites/ rid/Lists/BibliotecaDigital/RIDE/DE/DIJ/RESOLUCION-8430-DE-1993.PDF

Riveros Munévar, F., Bohórquez Borda, D., López Castillo, S., \& Sepúlveda Rodríguez, E. (2015). Diseño y Validación de un Instrumento para Medir las Actitudes frente a la Labor Profesional del Psicólogo. Revista Iberoamericana de Psicología, 8(2), 55-66. Obtenido de http://revistas. iberoamericana.edu.co/index.php/ripsicologia/article/view/814

Vallejo Giraldo, F. (2009). Informe del Proyecto Bienestar, Equidad y Permanencia Estudiantil - Bepes UDEA 2008-2010. Medellín [Co]: Universidad de Antioquia.

Vásquez Velásquez, J., Castaño Vélez, E., Gallón Gómez, S., \& Gómez Portilla, K. (2003). Determinantes de la Deserción Estudiantil en la Universidad de Antioquia (Informe Final de Investigación). Medellín [co]: Universidad de Antioquia. Obtenido de http://ciencias.udea.edu.co/ documentos/reacreditacion2/anexos/Anexo\%2039.\%20Estudio\%20 de\%20deserci\%C3\%B3n\%20estudiantil\%20en\%20la\%20Universidad/ Investigaci\%C3\%B3n\%20sobre\%20deserci\%C3\%B3n.pdf 